\title{
Hydrodynamic Studies of the Evolution of Recurrent Novae to Supernova Ia Explosions
}

\author{
S. Starrfield ${ }^{1}$, F. X Timmes ${ }^{1}$, W. R. Hix ${ }^{2}$, C. Iliadis ${ }^{3}$, W. D. Arnett ${ }^{4}$, \\ C. Meakin ${ }^{5}, \&$ W. M. Sparks ${ }^{5}$ \\ ${ }^{1}$ School of Earth and Space Exploration, Arizona State University, Tempe, AZ 85287-1404, \\ email: starrfield@asu.edu; fxt44@mac.com \\ ${ }^{2}$ Dept. of Physics and Astronomy, University of Tennessee, Knoxville, TN 37996-1200 \\ email: raph@utk.edu \\ ${ }^{3}$ Dept. of Physics \& Astronomy, University of North Carolina, Chapel Hill, NC 27599-3255, \\ email:iliadis@unc.edu \\ ${ }^{4}$ Dept. of Astronomy, University of Arizona, Tucson, AZ, 85721; \\ email:darnett@as .arizona.edu \\ ${ }^{5}$ Los Alamos National Laboratory, Los Alamos, NM, 87545; \\ email:casey.meakin@gmail.com; warrensparks@comcast.net
}

\begin{abstract}
We have begun new studies of the evolution of thermonuclear runaways (TNRs) in the accreted envelopes of white dwarfs (WDs). Here we focus on the recent outbursts of RS Oph (2006), U Sco (2010) and T Pyx (2011). U Sco explodes about every 10 years and the ejected material from the WD is helium rich. It has a short orbital period for recurrent novae $(\mathrm{RNe})$ but the secondary is likely to be evolved. The WD is thought to be close in mass to the Chandrasekhar limit. T Pyx has just suffered its first outburst since 1966 and it was predicted to never experience another outburst. It has a short orbital period and has formed dust in the ejecta as this paper was being written. One important question is the secular evolution of the WD. Do the repeated outbursts cause the WD to gain or lose mass? If it is gaining mass, it could eventually reach the Chandrasekhar limit and become a Type Ia supernova (SNe Ia) if it can hide the hydrogen and helium in the system. Here, we report on our latest studies of TNRs in accreted envelopes on WDs using a variety of initial WD masses, luminosities, and mass accretion rates. Of great importance to our conclusions, we assume a solar composition (Lodders abundance distribution). We use our 1-D hydro code, NOVA, that includes the Hix and Thielemann nuclear reaction network, the Iliadis reaction rate library, the Timmes equation of state, OPAL opacities, and the new convection of Arnett, Meakin, and Young. We report on the amount of ejected mass, evolution time to explode, and whether or not the WD is growing or losing mass.
\end{abstract}

Keywords. stars: white dwarfs, close binaries, dwarf novae, interiors, novae, cataclysmic variables, supernovae

\section{Introduction}

SNe Ia are of importance both to our understanding of the evolution of the Universe and to the Galactic chemical evolution of the iron group elements. Nevertheless, there is no general agreement on the progenitors of the explosion. The two major ideas are the single degenerate and double degenerate scenarios. In the standard paradigm single degenerate (SD) scenario, it is proposed that a WD in a close binary system accretes material from its companion and grows to the Chandrasekhar limit. As it nears the limit, an explosion is initiated in the core. In contrast, the double degenerate (DD) scenario requires the merger or collision of two WDs to produce the observed explosion. While for 
many years the SD scenario was the more prominent, a number of concerns have now led to major efforts to better understand the DD scenario, in spite of the fact that the SD scenario is capable of explaining most of the observed properties of the SNe Ia explosions via the delayed detonation model (Woosley \& Kasen 2011, and references therein).

In this paper we explore the SD scenario for SNe Ia progenitors which is based on the suggestion of Whelan and Iben (1973) that the outburst occurs in a close binary system that contains a WD and another star. Since the WD is accreting material from a secondary, virtually every type of close binary has been suggested as a SN Ia progenitor but one of the defining characteristics of a SN Ia explosion is the absence of hydrogen or helium in the spectrum at any time during the outburst or decline. This absence rules out most of the proposed close binary progenitors. A recent and detailed review of SNe Ia can be found in Howell (2011).

In support of the SD scenario, observations of V445 Pup (Nova 2000) imply that it was a helium nova (helium accretion onto a WD) because there were no signs of hydrogen in the spectrum at any time during the outburst but there were strong lines of carbon, helium, and other elements (Woudt et al. 2009 and references therein). The secondary is thought to be a hydrogen deficient carbon star (Woudt et al. 2009). Its existence, therefore, implies that there are binaries in which hydrogen is absent. Further motivation is that there are new observational searches for faint SNe Ia or other objects that might fill the "gap" in brightness between SNe Ia and bright classical novae (Kasliwal, these proceedings).

\section{The Nova Code}

We use our one-dimensional (1-D) hydrodynamic computer code (NOVA) to study the accretion of solar composition material onto WDs with masses of $0.4 \mathrm{M}_{\odot}, 0.7 \mathrm{M}_{\odot}, 1.0 \mathrm{M}_{\odot}$, $1.25 \mathrm{M}_{\odot}$, and $1.35 \mathrm{M}_{\odot}$. We use two initial WD luminosities $\left(4 \times 10^{-3} \mathrm{~L}_{\odot}\right.$ and $\left.10^{-2} \mathrm{~L}_{\odot}\right)$ and seven mass accretion rates ranging from $2 \times 10^{-11} \mathrm{M}_{\odot} \mathrm{yr}^{-1}$ to $2 \times 10^{-6} \mathrm{M}_{\odot} \mathrm{yr}^{-1}$. Our initial conditions are chosen to mimic those observed for the broad variety of CVs just as we have done in our previous studies of the classical and recurrent nova outbursts. We use the updated version of NOVA (Starrfield et al. 2009) that includes a nuclear reaction network that has now been extended to 187 nuclei (up to ${ }^{64} \mathrm{Ge}$ ). We also use the latest nuclear reaction rate compilation of Iliadis and collaborators that allow us to determine some of the uncertainties in the calculations (Iliadis et al. 2010). NOVA includes the latest microphysics (equations of state, opacities, and electron conduction) and the version of mixing-length convection theory as formulated by Arnett, Meakin, \& Young (2010). We find that using the updated code produces quantitative but not qualitative changes in our classical nova simulations (Starrfield et al. 2011, in prep.). These new simulations with NOVA were done with 150 Lagrangian zones and a surface zone mass reduced to $\sim 10^{-9} \mathrm{M}_{\odot}$.

\section{Motivation}

In addition to studying the consequences of accretion at a variety of rates onto a variety of WD masses, we are interested in comparing our simulations to earlier results shown in the $\dot{\mathrm{M}}-\mathrm{M}_{\mathrm{WD}}$ plane usually attributed to Fujimoto (1982a,b) and Nomoto (1982). A version of this plot is given as Figure 5 of Kahabka \& van den Heuvel (1997). This plot, in various forms, was shown many times at this symposium and we do not reproduce it here. This plot has 3 regions on it. For the lowest mass accretion rates, at all WD masses, accretion results in flashes which are normally expected to resemble those of the 
classical novae (Starrfield, Iliadis, \& Hix 2008). For the highest mass accretion rates, it is expected that the radius of the WD rapidly expands to red giant dimensions. There is a third predicted regime, intermediate between these two, where the material is supposed to steadily burn at the accretion rate. This $\dot{\mathrm{M}}$ is nominally $\sim 3 \times 10^{-7} \mathrm{M}_{\odot} \mathrm{yr}^{-1}$ and has a slight variation with WD mass. The implications of this plot is that most of the observed systems are accreting at rates that are not within the "steady burning" regime and, therefore, the WDs cannot be growing in mass. It is also assumed that those systems that are accreting at the "steady burning" rate are evolving horizontally towards higher WD mass and, presumably, are stuck in this mass accretion range. The supersoft sources in the LMC have been predicted to be in the "steady burning" regime (Kahabka \& van den Heuvel 1997, and references therein). A corollary to this scenario is that most systems which contain a WD cannot be SNe Ia progenitors. It is important to test this picture. We emphasize, however, that this plot implies that the only parameters that affect the evolution of a WD are its mass and $\dot{M}$. A priori it assumes nothing about the chemical composition of the accreting material, the chemical composition of the underlying WD, or if mixing of accreted material with core material has taken place. In addition, it assumes nothing about the thermal structure of the underlying WD and the effects of previous (or continuing) outbursts on the thermal and compositional structure of the WD. It has long been known that all these parameters affect the evolution of the WD (Starrfield 1989).

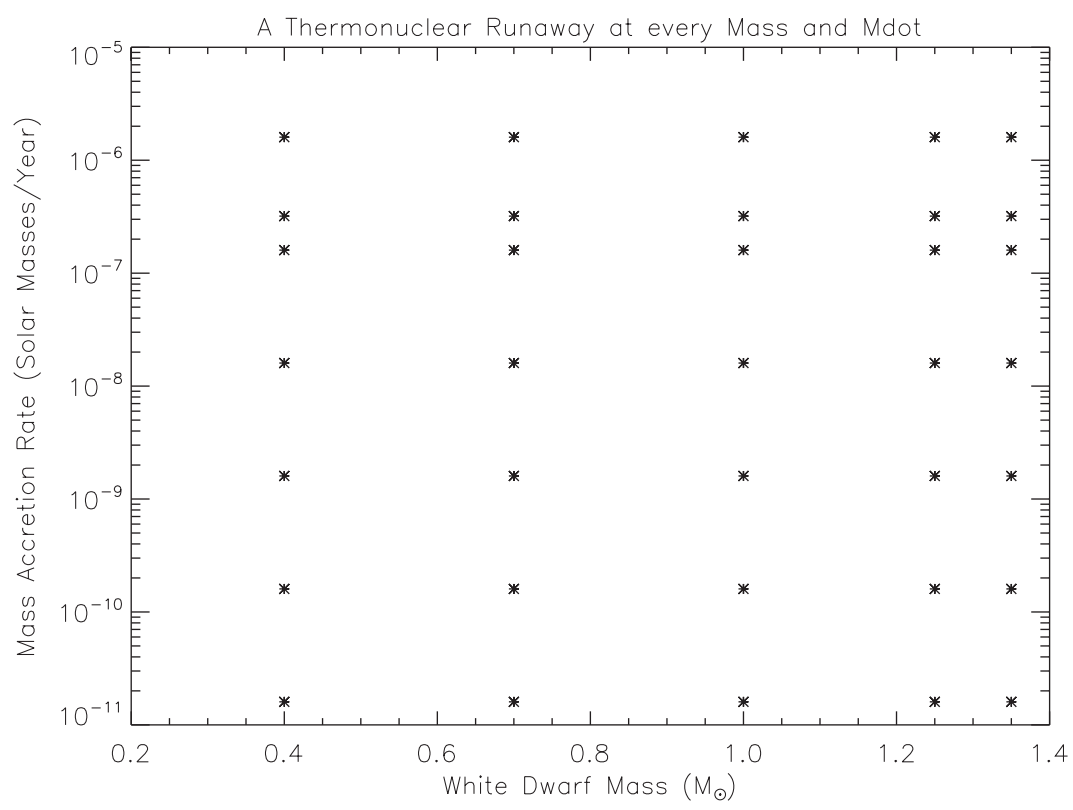

Figure 1. This plot shows each of the 70 evolutionary sequences that we calculated for this paper. Each of these simulations exhibited a TNR. In no case did "steady burning" occur.

Therefore, we can test the assumptions and predictions of this plot (and the underlying studies upon which it is based), simply by accreting solar material onto the WD, assume no mixing has occurred, and that this is the "first" outburst on the WD. In later studies we will relax some of these assumptions but our fundamental result is that the basic results summarized in this plot are not correct. 


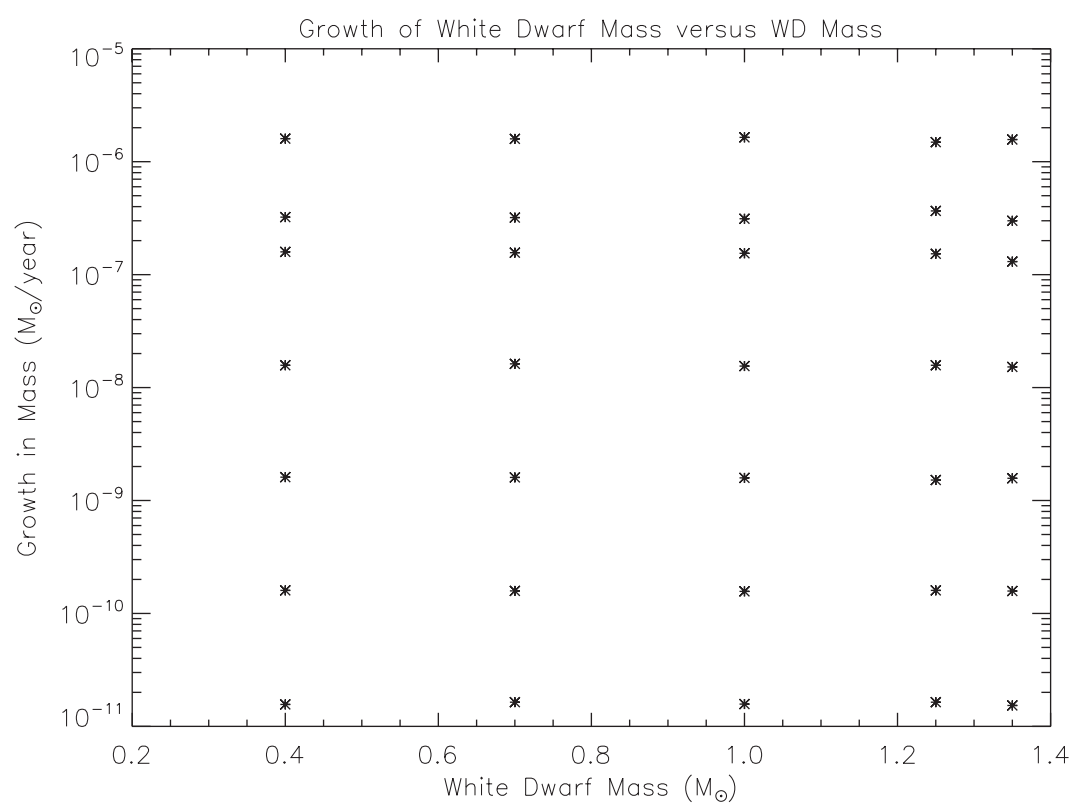

Figure 2. Here we plot the difference between the mass accreted and the mass lost. We display these results as the growth in mass $\left(\mathrm{M}_{\odot} \mathrm{yr}^{-1}\right)$ as a function of WD mass for each of our simulations.

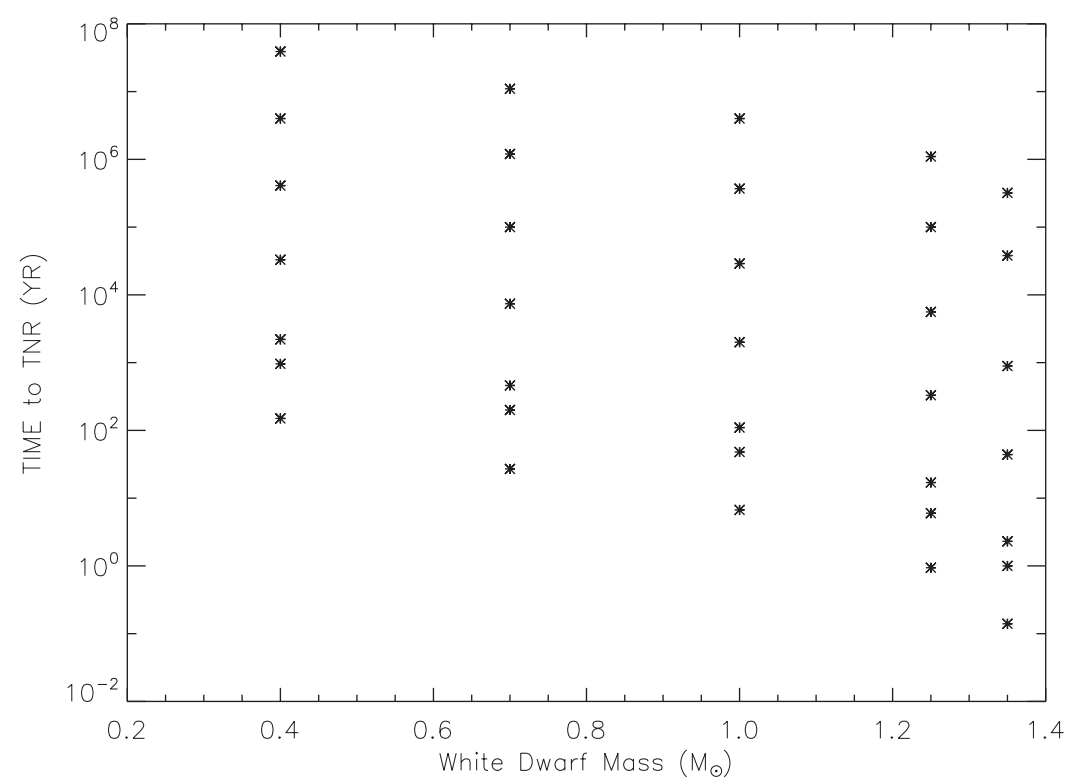

Figure 3. This is a plot of the accretion time to the TNR as a function of WD mass. The accretion time, for a given $\dot{M}$, decreases with WD mass because it takes less mass to initiate the TNR as the WD mass increases.

\section{Results}

Figure 1 shows the results for all 70 simulations that we have done (each data point represents two initial luminosities). In all cases we obtain a TNR which can eject some material, and after some evolutionary time, may cause the radius of the WD to grow. In 


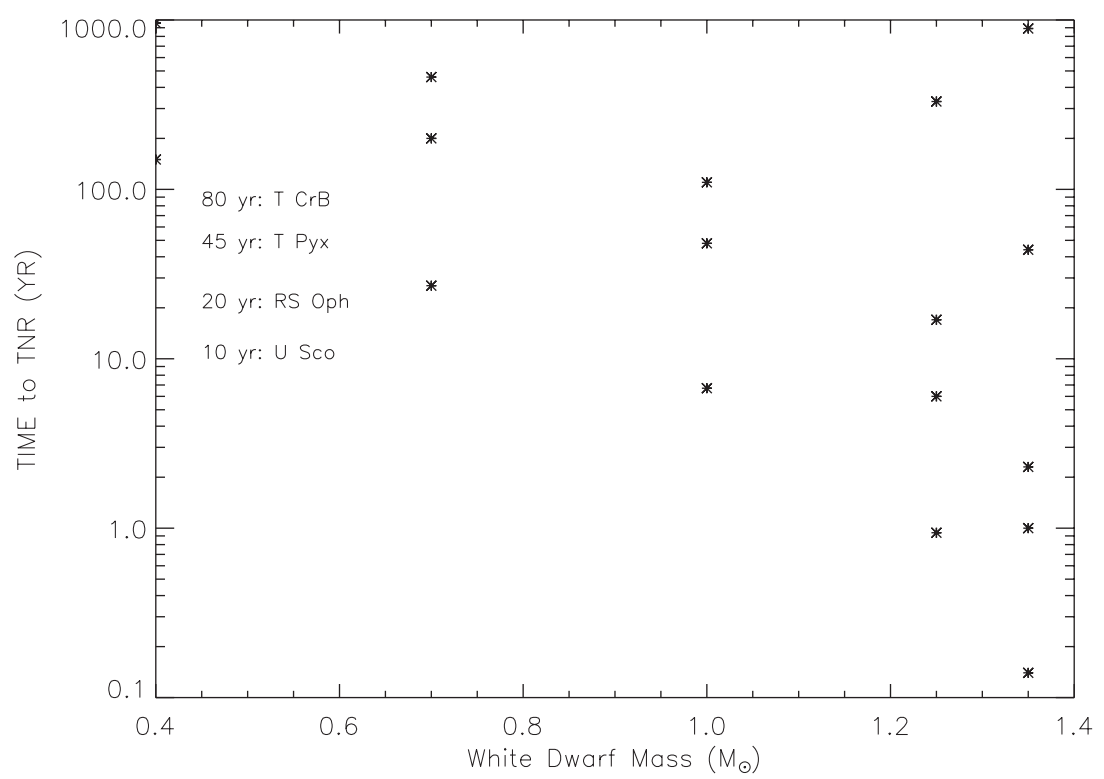

Figure 4. This is the same plot as Figure 3 but here we concentrate on the lower right corner and add approximate recurrence times for the best known RNe. This plot shows that not only is it possible for RNe outbursts to occur on low mass WDs but they can also occur for a broad range of $\dot{M}$ on higher mass WDs.

no case does "steady burning" as described in the literature occur. These fully timedependent calculations shows that the sequences exhibit the Schwarzschild \& Härm (1965) thin shell instability which precludes "steady burning." We also find that low mass WDs do not eject any mass while the high mass WDs do eject a small fraction of the accreted material. The most important result from our study is shown in Figure 2 where we plot the growth in mass versus WD mass for each of the simulations. We find that all these WDs are growing in mass as a result of the accretion of solar material. This is, as well known, not the case for classical novae which show core material in their ejecta and the WD must be losing mass as a result of the outburst. We identify these systems with those recurrent novae that do not show core material in their ejecta. It is also likely that for typical cataclysmic variables (dwarf novae and related objects) that the WD is growing in mass. This may explain the results shown by Zorotovic et al. (2011). Because of lack of space, we do not display the plot which shows which of these sequences is losing mass and how much. However, in Figure 2, we show the results of mass accreted minus mass lost as a function of WD mass for each sequence.

Finally, we also tabulated the accretion time to TNR for all our sequences and show those results in Figure 3. Clearly, as WD mass increases, the accretion time decreases for the same mass accretion rate. This is well known and a result of that fact that higher mass WDs reach the TNR with a smaller amount of accreted mass. In Figure 4, we concentrate on the lower right corner of Figure 3 and add approximate recurrence times for the best known RNe. Although it is often claimed that only the most massive WDs have recurrence times short enough to agree with these RNe, this plot shows that is not the case. It is possible for some of these RNe to occur on WDs with masses as low as $0.7 \mathrm{M}_{\odot}$. Therefore, basing "masses" of these RNe on short recurrence times is incorrect. We also note that it is possible for a RNe outburst to occur on a high mass WD for an extremely broad range of $\dot{M}$. A clue to the WD mass is the X-ray emission at maximum 
since it is likely that X-ray emission observed at maximum can only occur on massive WDs. We will show additional results elsewhere along with more implications.

We gratefully acknowledge partial support from the U. S. National Science Foundation, NASA, and the DOE.

\section{References}

Arnett, W. D., Meakin, C., \& Young, P. A. 2010, ApJ, 710, 1619

Fujimoto, M. Y. 1982a, ApJ, 257, 752

Fujimoto, M. Y. 1982b, ApJ, 257, 767

Howell, D. A. 2011, Nature Comm., 2, 350

Iliadis, C., et al. 2010, Nuclear Physics A, 841, 31

Kahabka, P. \& van den Heuvel, E. P. J. 1997, ARAA, 35, 69

Nomoto, K. 1982, ApJ, 253, 798

Schwarzschild, M. \& Härm, R. 1965, ApJ, 142, 855

Starrfield, S. 1989, in Classical Novae, ed. M. Bode \& A. Evans, Wiley, 39

Starrfield, S., Iliadis, C., \& Hix, W. R. 2008, "Thermonuclear Processes," in Classical Novae II, ed. M. Bode \& A. Evans, Cambridge University Press, 77

Starrfield, S., Iliadis, C., Hix, W. R., Timmes, F. X., \& Sparks, W. M. 2009, ApJ, 692, 1532

Whelan, J. \& Iben, I. 1973, ApJ, 186, 1007

Woosley, S. E. \& Kasen, D. 2011, ApJ, 734, 38

Woudt, P. A., et al. 2009, ApJ, 706, 738

Zorotovic, M., Schreiber, M. R., \& Gänsicke, B. T. 2011, A\&A, 536, 42 